\title{
sciendo
}

Current Issues in Pharmacy and Medical Sciences

Formerly ANNALES UNIVERSITATIS MARIAE CURIE-SKLODOWSKA, SECTIO DDD, PHARMACIA

journal homepage: http://www.curipms.umlub.pl/

\section{Investigation of excipients influence on rheological behaviour of hydrogels with dimethindene maleate and dexpanthenol: conditions of controlled shear rate}

\author{
Tetiana V. Popova ${ }^{1} \oplus$ Halyna P. Kukhtenko $^{1 \star}{ }^{\circledR}$, Ievgenit V. Gladukh ${ }^{1}$, \\ Oleksandr S. Kukhtenko ${ }^{1}$, Anzhela B. Olkhovska ${ }^{2}$ (i) \\ ${ }^{1}$ Department of Technologies of Pharmaceutical Preparations, National University of Pharmacy, Kharkiv, Ukraine \\ ${ }^{2}$ Department of Pharmaceutical Management and Marketing, National University of Pharmacy, Kharkiv, Ukraine
}

\begin{tabular}{l}
\hline ARTICLE INFO \\
\hline Received 12 April 2021 \\
Accepted 10 May 2021 \\
\hline Keywords: \\
hydrogel, \\
dimethindene maleate, \\
dexpanthenol, \\
hydroxypropyl methylcellulose \\
(HPMC), \\
xanthan gum, \\
carbomer, \\
rheology, \\
thixotropy, \\
rheopecty.
\end{tabular}

\begin{abstract}
Every year, the number of cases of hypersensitivity to insect bites increases. Thus, particular attention needs to be paid to the treatment of insect allergy in children, as scratching the bites can complicate the healing process and lead to infection. Therefore, a topical issue for modern medicine and pharmacy is the development of a gel of combined anti-allergic, reparative and anti-inflammatory action for the treatment of local manifestations of allergic skin reactions. Rheological studies are one of the stages of pharmaceutical development of soft dosage forms. In this study, we perform rheological studies of hydrogels containing $0.1 \%$ dimethindene maleate and 3.0\% dexpanthenol made on different carriers of dispersed structures: Carbopol ${ }^{\text {ptx }}$ Polymers carbomer Ultrez $10 \mathrm{NF}$, Hydroxypropyl methylcellulose (HPMC) of brand Metolose SR-90SH-100000SR, Ziboxan F200 xanthan gum. The rheological studies were undertaken using a rheometer in controlled shear rate mode CSR. Basic structural and mechanical indices of the dispersed systems were determined. These included: yield stress, structural viscosity, viscosity at infinite shear rate, the hysteresis loop area. Moreover, dynamic liquefaction coefficients have been calculated. Based on the rheological studies of hydrogels containing $0.1 \%$ dimethindene maleate and $3.0 \%$ dexpanthenol prepared with various carriers, the use of $1.8 \%$ and $2.0 \%$ HPMC hydrogels, $2.0 \%$ and $2.5 \%$ xanthan gum hydrogels, $0.5 \%$ and $0.75 \%$ carbomer gels is recommended for further biopharmaceutical studies. The application of one of these formulations as the final composition, provides adhesion and will not complicate the scaling-up of the process.
\end{abstract}

\section{INTRODUCTION}

Particular attention is paid to the study of the rheological characteristics of semisolid medicines at the stages of composition and manufacturing technology development [1]. The main characteristics under study include: structural viscosity, yield stress, thixotropy, shear stress, the determination of which can serve as an effective and objective parameter for quality control by implementing the quality by design strategy. A rheological behavior is closely related to the dispersed structure of the product, and using it makes possible to determine the structural changes that are observed during mixing, heat treatment, transportation, dosing, storage and use [2]. The information obtained from rheological studies

\footnotetext{
* Corresponding author

e-mail: galinakukh@gmail.com
}

is of particular relevance during scaling-up and technology transfer from laboratory to industrial conditions. There are numerous rotational tests available today, but according to the mode of operation they are classified into the methods of "controlled shear rate CSR or CR" and "controlled shear stress CSS or CS". In this work, a rheometer of controlled shear rate was used [3].

The NUPh's Department of Technologies of Pharmaceuticals carried out scientific work on the development of a composition of a gel with dimethindene maleate and dexpanthenol for the treatment of contact allergy. This was done under the direction of 0114U000945 "Development of composition, technology and biopharmaceutical study of medicinal products based on natural and synthetic raw materials" $[4,5]$. According to the classification of medicinal products 
on the basis of their equivalence, the developed drug belongs to the group A.3 - an original medicinal product with a fixed combination. A new combination of known active ingredients is also considered an original drug [6].

The therapeutic dose of dimethindene maleate in the gel was $1 \mathrm{mg} / 1 \mathrm{~g}$, dexpanthenol $30 \mathrm{mg} / 1 \mathrm{~g}$. Dimethindene maleate, a derivative of phenylalkylamine, belongs to the group of antihistamines of the first generation and has anti-allergic and antipruritic therapeutic effect by blocking H1-receptors. Dimethindene maleate is available on the market in various dosage forms. In products of systemic action, the therapeutic dose is $1 \mathrm{mg} / 1 \mathrm{tab}$; in nasal forms $0.25-1 \mathrm{mg} / 1 \mathrm{ml}$; in products of local dermatological action $1 \mathrm{mg} / 1 \mathrm{~g}$ [7].

Dexpanthenol is otherwise known as provitamin B5. Its action is similar to that of pantothenic acid, but it is better absorbed when applied topically. Pantothenic acid is a component of coenzyme A, which in the form of coenzyme acetyl-CoA plays an important role in cellular metabolism. Pantothenic acid is necessary for the formation and regeneration of the skin and mucous membranes. Dexpanthenol is used in topical agents in a dosage of $30-50 \mathrm{mg} / 1 \mathrm{~g}$ [7].

This combination of the two active substances will provide a comprehensive therapeutic effect on the pathological process in the local treatment of allergic skin reactions to irritants such as insect bites, extensive sun erythema, uncomplicated small skin burns and allergic skin irritations of small area. One of the stages of pharmaceutical development of local action medicines is the substantiation of the active ingredients delivery system to the place of action, which is the main purpose of this work.

Today, there are various strategies for ensuring the effective delivery of skin medicines, both through careful selection of the base components and through manufacturing techniques [8-10]. Hydrogels and bioadhesive hydrogels have been considered by us as pharmaceutical systems for the delivery of dimethindene maleate and dexpanthenol to the focus of the inflammatory allergic process caused by the action of external stimuli, such as that described above. Hydrogels are three-dimensional, cross-linked grids of water-soluble polymers and consist of at least two components, one of which forms a continuous three-dimensional macromolecular grid acting as a framework, and the other is a low-molecular solvent (usually demineralized water) which is uniformly dispersed throughout the system. The ability of the polymer to create this grid depends on many factors: the structure of the molecule (length and number of linearly oriented sections, the presence of side chains), the presence of active or activated groups for the process of cross-linking, etc. Natural polysaccharides such as alginates, gums, pectins, methylcellulose and its derivatives or other natural and synthetic substances can be used as such substances [11]. It should be noted that they can form systems with the properties of either viscous-plastic fluid, viscous-plastic solid or solid (jelly). Therefore, the study of rheological behavior and the determination of structural and mechanical parameters at the stage of development of the composition of a drug in the form of a gel becomes particularly important [12].
A feature of bioadhesive hydrogels is the provision of a longer residence time at the application site, thereby maintaining a high local concentration of the active ingredient in the surrounding tissues over a long period, which is important for prolonged exposure to the inflammatory process and reduces the frequency of the drug use. This mechanism can be used to deliver active ingredients intended for both surface and resorptive action [13-15].

\section{MATERIALS AND METHODS}

Based on the analysis of the range of gel-forming substances for the development of the composition of the hydrogel containing $0.1 \%$ dimethindene maleate and $3.0 \%$ dexpanthenol, the following substances have been selected [7]: Hydroxypropyl methylcellulose (HPMC) of Metolose SR-90SH-100000SR brand (Ph. Eur. 9.2), manufactured by Shin-Etsu Chemical Co., Ltd, Japan; Xanthan gum Ziboxan F200 (Ph. Eur. 9.2), produced by Deosen Biochemical Ltd, China; Carbopol TM Polymers Ultrez 10 NF (USP32 - NF27) carbomer manufactured by Lubrizol Corp., USA.

The excipients listed are widely used in topical pharmaceuticals, and the rheological properties of the substances are well studied $[11,14,16]$. However, the introduction of active substances to pure hydrogels can significantly affect their rheological properties: structural viscosity, thixotropy, yield stress, etc., and at the same time may lead to the system's unsuitability to the technological process of production and use by patients [17].

The substances selected differ in their nature of origin and mechanism of gelation. Carbomers are a group of synthetic high molecular weight polymers of acrylic acid, crosslinked by allyl ether of sucrose or pentaerythritol [17]. The use of polyacrylic acid derivatives, according to the authors, provides bioadhesive properties of the drug $[16,18]$.

HPMC is a partially methylated and hydroxypropylated cellulose obtained from wood pulp (fibers) under controlled conditions $[16,17]$. Xanthan gum is an extracellular anionic polysaccharide produced by the microorganism Xanthomonas campestris. The polysaccharide is formed in the process of complex fermentation on the surface of their cell wall in the course of their normal life cycle [17].

Gel based samples have been produced containing $0.1 \%$ dimethindene maleate (Ph Eur 8.2; BASF SE, Germany), dexpanthenol 3.0\% (Ph Eur 8.2; BASF SE, Germany) and the preservative 2-phenoxyethanol 0.75\% (Ph Eur 8.2; Euxyl® PE 9010, "Schulke \& Mayr GmbH", Germany) $[3,7,19,20]$. Purified water (SPU 2.0) was used as the dispersion medium in all formulations. The technology of introduction of the active ingredients into the gel base was the same in all samples. The samples differed by the type of gelling agent used and its concentration.

The dimethindene maleate substance (N, N-dimethyl-2[3- (1-pyridin-2-ylethyl) -1H-inden-2-yl] ethanamine, CAS 5636-83-9) is a solid fine powder, slightly soluble in water (239 $\mathrm{mg} / \mathrm{L}\left(\right.$ at $\left.37 \pm 0.5^{\circ} \mathrm{C}\right)$ ), but well soluble in propylene glycol at a temperature of $40 \pm 0.5^{\circ} \mathrm{C}$. Dimethindene maleate was introduced into the gel base as solution, pre-dissolving it in propylene glycol in a ratio of $1: 100$. Substances of dexpanthenol (2R) -2,4-dihydroxy-N- (3-hydroxypropyl) 
-3,3-dimethylbutanamide, CAS 81-13-0; 7732-18-5) and 2-phenoxyethanol (CAS 122-99-6) are liquids with different viscosities, so the required amounts were added directly to the base of the gel.

The technology of active ingredients introduction to the gel base was the same in all samples: first dexpanthenol was introduced and homogenized, then propylene glycol solution of dimethindene maleate was added and homogenized, the last, the preservative 2-phenoxyethanol, was then added.

Gel bases without API (Active pharmaceutical ingredient) content were made with the addition of $10 \%$ propylene glycol.

\section{Method of HPMC hydrogels preparation}

For the preparation of HPMC hydrogels, 1/3 part of the water purified calculated from the formula was heated to $80^{\circ} \mathrm{C}$ and the polymer was added with stirring for $30 \mathrm{~min}$, then the rest of the purified water of room temperature was added. The concentration of HPMC in the test samples was $1.2 \% ; 1.6 \% ; 1.8 \%$ and $2.0 \%$. One hour after preparation of the base, the active ingredients were added.

\section{Method of xanthan gum hydrogels preparation}

Xanthan gum was added to the calculated amount of purified water at room temperature and stirred for $30 \mathrm{~min}$. Gel samples with xanthan gum content of $1.5 \%, 2.0 \%$, and $2.5 \%$ were made. One hour after preparation of the base, the active ingredients were added.

\section{Method of carbomer hydrogels preparation}

Carbomer was added to the calculated amount of water purified of room temperature, dispersed for 30 minutes, and then neutralized with trometamol solution $(40 \%)$ to the $\mathrm{pH}$ of the dispersed system $6.5 \pm 0.3$ [21,22]. Carbopol ${ }^{\mathrm{TM}}$ Ultrez $10 \mathrm{NF}$ carbomer was used in the work. In the production of these gel samples, we were guided by the technical documentation of the carbomer manufacturer. The concentration of carbomer in the samples was $0.5 \% ; 0.75 \%$ and $1.0 \%$. One hour after preparation of the base, the active ingredients were added.

Measurements of rheological properties of hydrogels were carried out one day after preparation according to the following method.

\section{Methods of rheological studies}

All measurements of rheological behavior and structuralmechanical parameters were performed using a Rheolab QC rotary rheometer manufactured by Anton Paar (Austria), with a C-CC27/SS cylindrical measuring system that complies with ISO 3219. The Rheolab QC rheometer was equipped with RheoPlus 32 V3.62 software, which allows to controlling the experiment conditions (temperature, shear rate range, number of measurement points and duration of one point measurement).

Using the RheoPlus 32 V3.62 software, the hysteresis loop area $(\mathrm{A}, \mathrm{Pa} / \mathrm{s})$ was calculated, as well as the point (stress) of the yield $\left(\tau_{0}, \mathrm{~Pa}\right)$, and the viscosity at infinite shear rate $\left(\eta_{\infty}, \mathrm{Pa} \cdot \mathrm{s}\right)$ using the mathematical model of Casson:

$$
\tau^{\frac{1}{p}}=\tau_{0}+\left(\eta_{\infty} \cdot \dot{\gamma}\right)^{\frac{1}{p}}
$$

where, $\tau_{0}$ - the yield stress, $\mathrm{Pa}$,

$\eta_{\infty}$ - viscosity at infinite shear rate by Casson, $\mathrm{Pa} \cdot \mathrm{s}$

$\dot{\gamma}$ - shear rate, $\mathrm{s}^{-1}$

$\mathrm{p}=2$ - regression parameter.

Casson's model describes a non-perfect plastic type of flow in which there is a disproportionate relationship between the shear rate and the stress and which most closely matches the nature of the flow of the hydrogels under study [23].

Measurements of rheological flow curves were performed in three stages:

a) a linear increase in the shear rate gradient from $0.1 \mathrm{~s}^{-1}$ up to $350 \mathrm{~s}^{-1}$ with 105 measurement points and duration of point measurement of $1 \mathrm{~s}$;

b) constant shear at a shear rate of $350 \mathrm{~s}^{-1}$, one measurement point of duration $1 \mathrm{~s}$;

c) linear decrease of the shear rate gradient from $350 \mathrm{~s}^{-1}$ to $0.1 \mathrm{~s}^{-1}$ with 105 measurement points and $1 \mathrm{~s}$ point measurement duration.

d) The shear rate gradient range is $0.1-350 \mathrm{~s}^{-1}$ corresponds to a speed range of 0.075-270 rpm.

The study of thixotropic properties was carried out in three stages:

a) constant shear at a speed $1 \mathrm{~s}^{-1}, 5$ measuring points, duration of point measuring $5 \mathrm{~s}$;

b) constant shear at a speed of $150 \mathrm{~s}^{-1}, 50$ measuring points, duration of point measuring $1 \mathrm{~s}$;

c) constant shear at speed $1 \mathrm{~s}^{-1}, 250$ measuring points, duration of point measuring $1 \mathrm{~s}$.

The study temperature of the rheological properties of the samples was $25 \pm 0.5^{\circ} \mathrm{C}$, each sample was thermostatically controlled for 20 minutes.

\section{Calculation of dynamic liquefaction coefficients}

The dynamic liquefaction coefficient was determined at shear rates of 3.4 and $10.3 \mathrm{~s}^{-1}$ corresponding to the shear rate of the palm when distributing a semisolid dosage form on the surface and the viscosity of the system at shear rates of 27.4 and $150 \mathrm{~s}^{-1}$, which corresponds to the speed of technological processing in the process of the gel manufacture $[3,24]$. On the basis of the obtained results, the values of the dynamic liquefaction coefficients of the system have been calculated by the formulas:

$$
\begin{gathered}
K_{d 1}=\frac{\eta_{3.4}-\eta_{10.3}}{\eta_{3.4}} \cdot 100 \% \\
K_{d 2}=\frac{\eta_{27.4}-\eta_{150}}{\eta_{27.4}} \cdot 100 \%
\end{gathered}
$$

where, $\mathrm{K}_{\mathrm{d} 1}$ and $\mathrm{K}_{\mathrm{d} 2}$ - dynamic liquefaction coefficients; $\eta$ is the structural viscosity at the corresponding shear rates, $\mathrm{Pa} \cdot \mathrm{s}$. 


\section{RESULTS}

Gelation is based on the process of continuous increase in the viscosity of the polymer solution in time, which is accompanied by an increase in viscoelastic properties and, depending on the concentration used, can lead to the formation of a resilient homogeneous non-flowing system - a jelly or gel. Conditionally, this process can be represented in two stages: the first stage is characterized by a significant increase in the viscosity of the system, but overall the system still retains the properties inherent in hydrocolloids; the second stage begins with the appearance of elasticity, that is, the beginning of the manifestation of the properties of a solid. However, the introduction of active substances into pure hydrogels can significantly affect their rheological properties, so this paper highlights the viscoelastic properties of gel bases and gels containing APIs.

In Figures 1.1, 1.2, experimental rheograms and calculated (theoretical) flow rheograms or flow behavior curves according to the mathematical Casson model of HPMC gel bases are presented, and in Figures 1.3, 1.4 the results of rheological studies of HPMC gels containing the active substances dimethindene maleate, dexpanthenol and 2-phenoxyethanol are displayed in logarithmic coordinates. Similar rheograms of xanthan gum gel bases and xanthan gum gels with API content are shown in Figures 2.1 and 2.2, while rheograms of carbomer gel bases and carbomer gels with API are presented in Figures 3.1 and 3.2. The graphs show the relationship between shear stress, structural viscosity and the shear rate in the controlled shear rate CSR under incremental increasing/decreasing in the shear rate gradient. In Figures. 1.5, 2.3, 3.3 the results of the study of thixotropy of hydrogels containing API in the conditions of rest - liquefaction - rest are shown. Tables 1.1, 2.1, 3.1 show selective values of shear stress and structural viscosity of gel bases and API-loaded gels in the incremental increasing/ decreasing of shear rate in the CSR controlled shear rate mode. The results of the calculated structural and mechanical parameters are given in Tables 1.2, 2.2, 3.2.

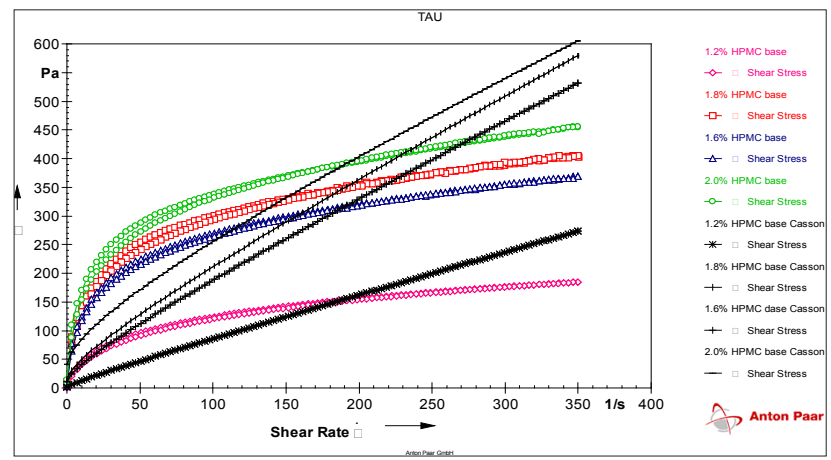

Figure 1.1 Experimental flow rheograms of HPMC gel bases and calculated (theoretical) flow rheograms according to the Casson model

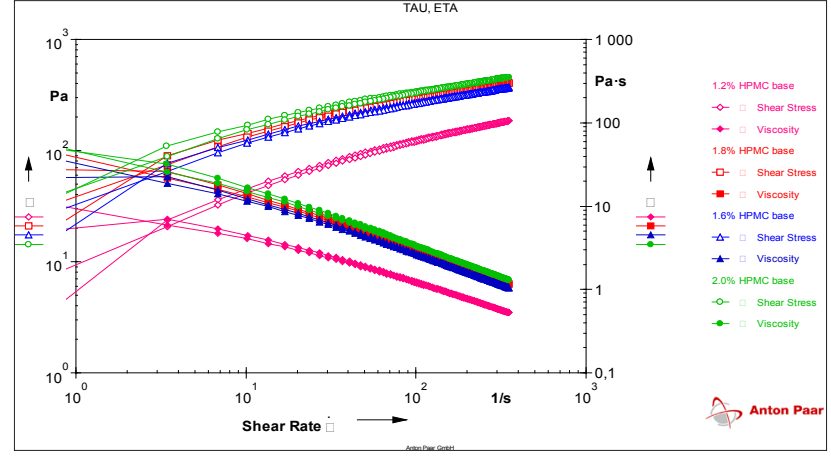

Figure 1.2 The dependence of the shear stress ( $\tau, \mathrm{Pa})$ of HPMC gel bases on the shear rate gradient $\left(, \mathrm{s}^{-1}\right)$ and the dependence of the structural viscosity $(\eta, \mathrm{Pa} \cdot s)$ on the shear rate gradient $\left(, \mathrm{s}^{-1}\right)$ - logarithmic coordinates $(\log )$

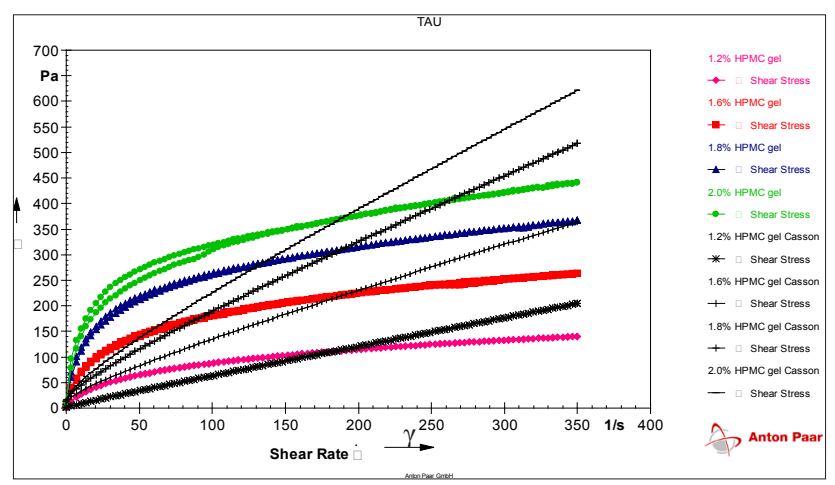

Figure 1.3 Experimental flow rheograms of HPMC gels containing API and calculated (theoretical) flow rheograms according to the Casson model

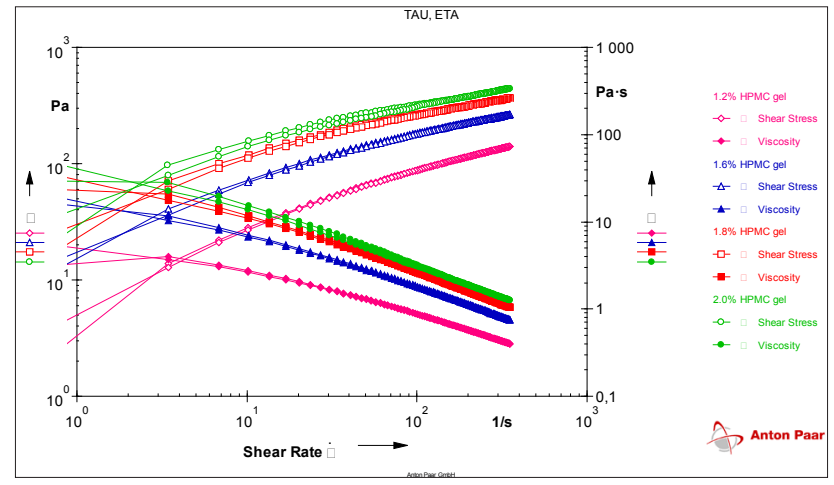

Figure 1.4 The dependence of the shear stress $(\tau, \mathrm{Pa})$ of HPMC gels with API content on the shear rate gradient $\left(\gamma, \mathrm{s}^{-1}\right)$ and the dependence of the structural viscosity $(\eta, \mathrm{Pa} \cdot s)$ on the shear rate gradient $\left(\gamma, \mathrm{s}^{-1}\right)$ - logarithmic coordinates $(\log )$

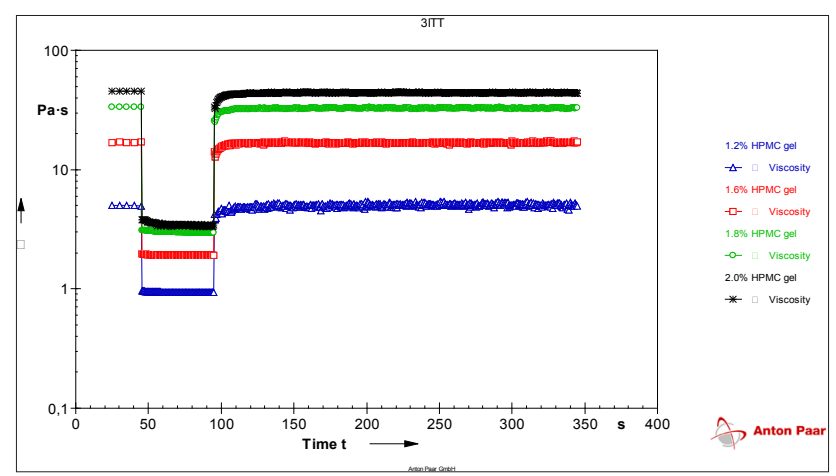

Figure 1.5 Dependence of structural viscosity $(\eta, \mathrm{Pa} \cdot \mathrm{s})$ of HPMC gels with API content on the shear rate gradient in the mode 1 $\mathrm{s}^{-1}-150 \mathrm{~s}^{-1}-1 \mathrm{~s}^{-1}$ 
Table 1.1. Values of shear stress and structural viscosity of HPMC gel bases and API-loaded HPMC gels in order of increasing/ decreasing shear rate gradient

\begin{tabular}{|c|c|c|c|c|}
\hline \multirow{2}{*}{$\begin{array}{l}\text { Shear rate gradient, } \\
\qquad\left(\dot{y}, \mathrm{~s}^{-1}\right)\end{array}$} & \multicolumn{4}{|c|}{ Gel bases } \\
\hline & $1.2 \% \mathrm{HPMC}$ & $1.6 \%$ HPMC & $1.8 \% \mathrm{HPMC}$ & 2.0\% HPMC \\
\hline \multicolumn{5}{|c|}{$\begin{array}{l}\text { The shear stress }(\mathrm{T}, \mathrm{Pa}) \text { at increasing/ } \\
\text { decreasing the shear rate gradient }\left(\dot{\mathrm{V}}, \mathrm{s}^{-1}\right)\end{array}$} \\
\hline 0.1 & $0.3 / 2.22$ & $2.04 / 9.35$ & $2.77 / 11.5$ & $8.83 / 13.7$ \\
\hline 3.4 & $23.9 / 20.6$ & $77.4 / 65$ & $89.6 / 74.2$ & $110 / 88.4$ \\
\hline 6.84 & $36.2 / 32.2$ & $107 / 96.2$ & $123 / 109$ & $148 / 128$ \\
\hline 10.3 & $45.3 / 10.2$ & $124 / 117$ & $143 / 132$ & $170 / 153$ \\
\hline 27.4 & $73.8 / 68.9$ & $184 / 175$ & $207 / 196$ & $241 / 223$ \\
\hline 150 & $143 / 140$ & $300 / 295$ & $331 / 328$ & $370 / 368$ \\
\hline \multicolumn{5}{|c|}{$\begin{array}{c}\text { Structural viscosity }(n, \mathrm{~Pa} \cdot \mathbf{s}) \text { at increasing/ } \\
\text { decreasing shear rate gradient }\left(\dot{\mathrm{y}}, \mathrm{s}^{-1}\right)\end{array}$} \\
\hline 0.1 & $3.42 / 21.2$ & $21.3 / 89.7$ & $29.2 / 111$ & $86.4 / 132$ \\
\hline 3.4 & $6.91 / 5.84$ & $22.5 / 18.8$ & $26.1 / 21.5$ & $31.9 / 25.6$ \\
\hline 6.84 & $5.3 / 4.72$ & $15.6 / 14.1$ & $18 / 15.9$ & $21.5 / 18.7$ \\
\hline 10.3 & $4.44 / 4.05$ & $12.2 / 11.5$ & $14 / 13$ & $16.7 / 15.1$ \\
\hline 27.4 & $2.73 / 2.55$ & $6.8 / 6.48$ & $7.67 / 7.26$ & $8.92 / 8.26$ \\
\hline 150 & $0.94 / 0.92$ & $1.98 / 1.95$ & $2.18 / 2.16$ & $2.44 / 2.43$ \\
\hline \multicolumn{5}{|c|}{ API-loaded gels } \\
\hline & $1.2 \% \mathrm{HPMC}$ & $1.6 \% \mathrm{HPMC}$ & $1.8 \% \mathrm{HPMC}$ & $2.0 \% \mathrm{HPMC}$ \\
\hline 0.1 & $0.2 / 0.9$ & $2.5 / 4.6$ & $2.7 / 8.5$ & $2.9 / 12.2$ \\
\hline 3.4 & $13.8 / 12.7$ & $40.5 / 35.9$ & $71.1 / 60.5$ & $69.8 / 78.8$ \\
\hline 6.84 & $21.9 / 20.9$ & $58.9 / 54.7$ & $100.0 / 91.0$ & $133.0 / 115.0$ \\
\hline 10.3 & $28.1 / 26.9$ & $71.8 / 68.7$ & 119.0/112.0 & $156.0 / 140.0$ \\
\hline 27.4. & $48.4 / 47.5$ & $114.0 / 109.0$ & $179.0 / 171.0$ & $227.0 / 206.0$ \\
\hline 150 & $105.0 / 104.0$ & 209.0/208.0 & $295.0 / 294.0$ & $352.0 / 351.0$ \\
\hline \multicolumn{5}{|c|}{$\begin{array}{c}\text { Structural viscosity }(\eta, P a \cdot s) \text { at increasing/ } \\
\text { decreasing shear rate gradient }\left(\dot{\hat{y}}, s^{-1}\right)\end{array}$} \\
\hline 0.1 & $2.3 / 8.7$ & $24.6 / 44.0$ & $27.8 / 81.6$ & $30.6 / 118.0$ \\
\hline 3.4 & $3.9 / 3.7$ & $11.7 / 10.4$ & $20.7 / 17.5$ & $28.2 / 22.8$ \\
\hline 6.84 & $3.2 / 3.1$ & $8.6 / 8.1$ & $14.7 / 13.3$ & $19.4 / 16.9$ \\
\hline 10.3 & $2.6 / 2.6$ & $7.1 / 6.7$ & $11.7 / 11.0$ & $15.3 / 13.7$ \\
\hline 27.4. & $1.8 / 1.8$ & $4.2 / 4.1$ & $6.6 / 6.3$ & $8.4 / 7.6$ \\
\hline 150 & $0.7 / 0.7$ & $1.4 / 1.3$ & $1.9 / 1.9$ & $2.3 / 2.3$ \\
\hline
\end{tabular}

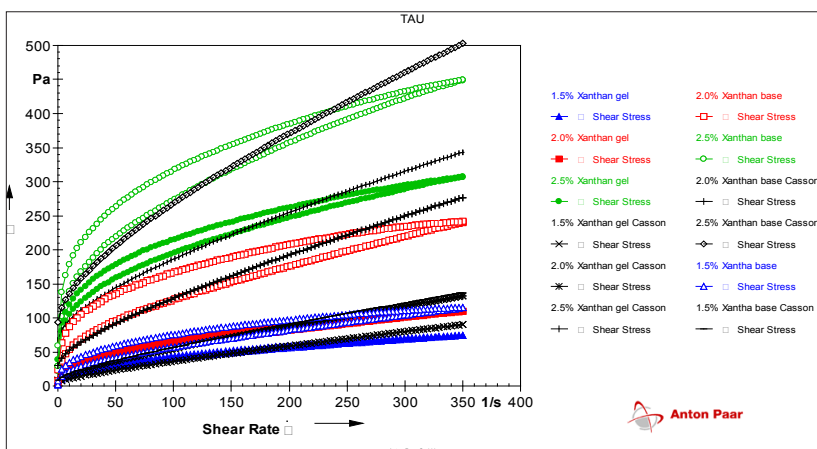

Figure 2.1. Experimental flow rheograms of xanthan gel bases and xanthan gels with API content and calculated (theoretical) flow rheograms according to the Casson model
Table 1.2. Calculated Structural-mechanical parameters of HPMC gel bases and API-loaded HPMC gels

\begin{tabular}{|l|c|c|c|c|}
\hline \multirow{2}{*}{ Indicators } & \multicolumn{4}{|c|}{ Gel bases } \\
\cline { 2 - 5 } & $1.2 \% \mathrm{HPMC}$ & $1.6 \% \mathrm{HPMC}$ & $1.8 \% \mathrm{HPMC}$ & $2.0 \% \mathrm{HPMC}$ \\
\hline $\begin{array}{l}\text { Hysteresis loop } \\
\text { area, Pa/s }\end{array}$ & 855.47 & 1176.65 & 1463.68 & 1334.56 \\
\hline $\begin{array}{l}\text { The yield stress by } \\
\text { Casson } \mathrm{T}_{0}, \mathrm{~Pa}\end{array}$ & 0.81 & 10.79 & 15.69 & 26.87 \\
\hline $\begin{array}{l}\text { Viscosity at infinite } \\
\text { shear rate by } \\
\text { Casson } \mathrm{\eta}_{\infty}, \mathrm{Pa} \cdot \mathrm{s}\end{array}$ & 0.69 & 1.15 & 1.21 & 1.98 \\
\hline $\begin{array}{l}\text { Dynamic } \\
\text { liquefaction } \\
\text { coefficient } \mathrm{K}_{\mathrm{d} 1}, \%\end{array}$ & 35.75 & 45.78 & 46.36 & 47.65 \\
\hline $\begin{array}{l}\text { Dynamic } \\
\text { liquefaction } \\
\text { coefficient } \mathrm{K}_{\mathrm{d} 2}, \%\end{array}$ & 65.57 & 70.88 & 71.58 & 72.65 \\
\hline & $1.2 \% \mathrm{HPMC}$ & $1.6 \% \mathrm{HPMC}$ & $1.8 \% \mathrm{HPMC}$ & $2.0 \% \mathrm{HPMC}$ \\
\hline $\begin{array}{l}\text { Hysteresis loop } \\
\text { area, Pa/s }\end{array}$ & 193.95 & 310.01 & 626.64 & 2239.37 \\
\hline $\begin{array}{l}\text { The yield stress by } \\
\text { Casson } \mathrm{T}_{0}, \mathrm{~Pa}\end{array}$ & 0.45 & 9.22 & 11.77 & 13.38 \\
\hline $\begin{array}{l}\text { Viscosity at infinite } \\
\text { shear rate by } \\
\text { Casson } \mathrm{\eta}_{\infty}, \mathrm{Pa} \cdot \mathrm{s}\end{array}$ & 0.53 & 0.74 & 1.07 & 1.29 \\
\hline $\begin{array}{l}\text { Dynamic } \\
\text { liquefaction } \\
\text { coefficient } \mathrm{K}_{\mathrm{d} 1}, \%\end{array}$ & 30.90 & 39.83 & 43.48 & 45.75 \\
\hline $\begin{array}{l}\text { Dynamic } \\
\text { liquefaction } \\
\text { coefficient } \mathrm{K}_{\mathrm{d} 2}, \%\end{array}$ & 61.73 & 67.93 & 71.10 & 72.98 \\
\hline
\end{tabular}

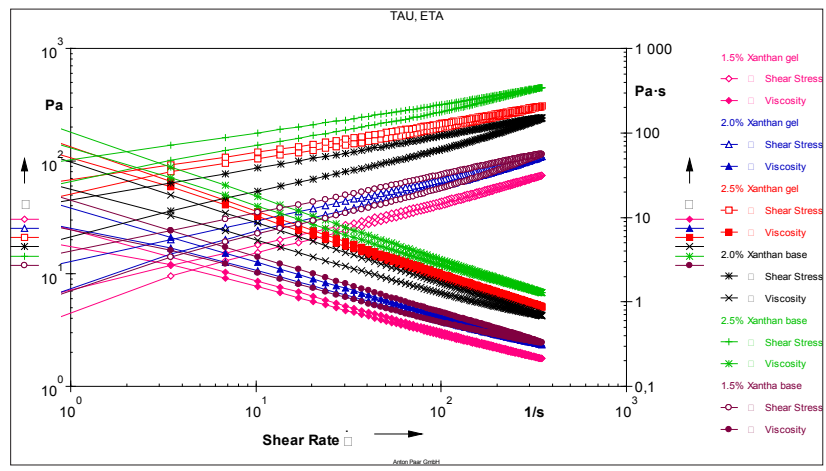

Figure 2.2. Dependence of shear stress $(\tau, \mathrm{Pa})$ of xanthan gel bases and xanthan gels with API content on shear rate gradient $\left(\dot{\gamma}, \mathrm{s}^{-1}\right)$ and dependence of structural viscosity $(\eta, \mathrm{Pa} \cdot s)$ on shear rate gradient $\left(\dot{\gamma}, \mathrm{s}^{-1}\right)$ - logarithmic coordinates $(\log )$

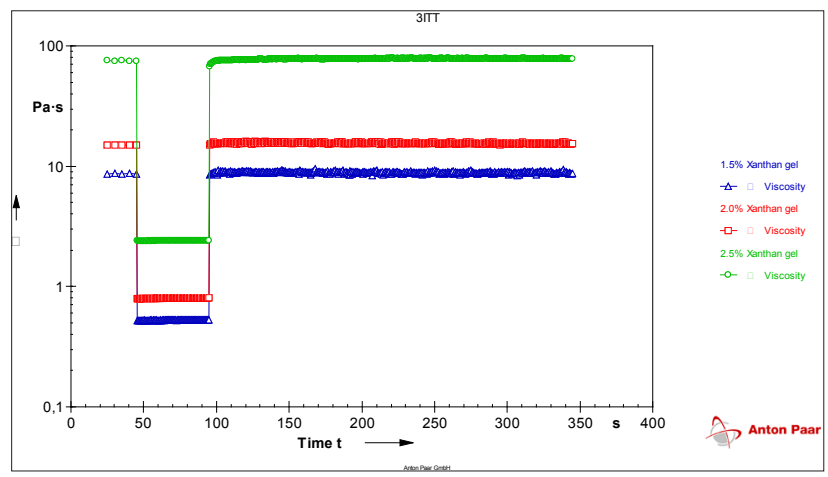

Figure 2.3. Dependence of structural viscosity $(\eta, \mathrm{Pa} \cdot \mathrm{s}$ ) of xanthan gum gels with API content on the shear rate gradient in the mode $1 \mathrm{~s}^{-1}-150 \mathrm{~s}^{-1}-1 \mathrm{~s}^{-1}$ 
Investigation of excipients influence on rheological behaviour of hydrogels with dimethindene maleate and dexpanthenol: ...

Table 2.1. Value of shear stress and structural viscosity of Xanthan gum gel bases and API-loaded gels in order of increasing/ decreasing shear rate gradient

\begin{tabular}{|c|c|c|c|}
\hline \multirow{2}{*}{$\begin{array}{l}\text { Shear rate gradient, } \\
\qquad\left(\dot{\mathrm{y}}, \mathrm{s}^{-1}\right)\end{array}$} & \multicolumn{3}{|c|}{ Gel bases } \\
\hline & $1.5 \%$ xanthan & $2.0 \%$ xanthan & $2.5 \%$ xanthan \\
\hline \multicolumn{4}{|c|}{$\begin{array}{l}\text { The shear stress }(\boldsymbol{T}, \mathrm{Pa}) \text { at increasing/ } \\
\text { decreasing the shear rate gradient }\left(\dot{\mathrm{Y}}, \mathrm{s}^{-1}\right)\end{array}$} \\
\hline 0.1 & $1.92 / 7.37$ & $7.86 / 23.6$ & $29.2 / 59.4$ \\
\hline 3.4 & $14 / 24.3$ & $36.2 / 63.8$ & $102 / 138$ \\
\hline 6.84 & $19.2 / 30.2$ & $47.2 / 77.1$ & $125 / 162$ \\
\hline 10.3 & $22.6 / 34.7$ & $54.2 / 86.6$ & $139 / 178$ \\
\hline 27.4 & $32.8 / 47.8$ & $76.6 / 113$ & $182 / 225$ \\
\hline 150 & $70.4 / 87.8$ & $153 / 190$ & $319 / 355$ \\
\hline \multicolumn{4}{|c|}{$\begin{array}{l}\text { Structural viscosity }(n, \mathrm{~Pa} \cdot \mathrm{s}) \text { at increasing/ } \\
\text { decreasing shear rate gradient }\left(\dot{\mathrm{y}}, \mathrm{s}^{-1}\right)\end{array}$} \\
\hline 0.1 & $20.2 / 73.1$ & $82.7 / 235$ & $301 / 589$ \\
\hline 3.4 & $4.06 / 7.0$ & $10.5 / 18.4$ & $29.5 / 39.7$ \\
\hline 6.84 & $8.81 / 4.42$ & $6.91 / 11.3$ & $18.3 / 23.8$ \\
\hline 10.3 & $2.21 / 3.41$ & $5.31 / 8.5$ & $13.6 / 17.5$ \\
\hline 27.4 & $1.21 / 1.77$ & $2.83 / 4.2$ & $6.73 / 8.34$ \\
\hline \multirow[t]{3}{*}{150} & $0.47 / 0.58$ & $1.01 / 1.26$ & $2.11 / 2.34$ \\
\hline & \multicolumn{3}{|c|}{ API-loaded gels } \\
\hline & $1.5 \%$ xanthan & $2.0 \%$ xanthan & $2.5 \%$ xanthan \\
\hline \multicolumn{4}{|c|}{$\begin{array}{l}\text { The shear stress }(\mathrm{T}, \mathrm{Pa}) \text { at increasing/ } \\
\text { decreasing the shear rate gradient }\left(\dot{\mathrm{r}}, \mathrm{s}^{-1}\right)\end{array}$} \\
\hline 0.1 & $1.1 / 2.9$ & $1.9 / 5.6$ & $21.1 / 38.7$ \\
\hline 3.4 & $9.6 / 11.8$ & $15.0 / 20.1$ & $80.2 / 92.0$ \\
\hline 6.84 & $13.0 / 15.2$ & $20.1 / 25.6$ & $69.0 / 109.0$ \\
\hline 10.3 & $15.5 / 17.9$ & $24.0 / 29.7$ & $105.0 / 120.0$ \\
\hline 27.4. & $23.0 / 26.2$ & $35.0 / 41.9$ & $134.0 / 152.0$ \\
\hline 150 & $49.2 / 52.3$ & $72.1 / 80.9$ & $225.0 / 243.0$ \\
\hline \multicolumn{4}{|c|}{$\begin{array}{c}\text { Structural viscosity }(n, P a \cdot s) \text { at increasing/ } \\
\text { decreasing shear rate gradient }\left(\dot{\gamma}, s^{-1}\right)\end{array}$} \\
\hline 0.1 & $11.1 / 28.4$ & $20.3 / 56.1$ & $232.0 / 385.0$ \\
\hline 3.4 & $2.8 / 3.4$ & $4.3 / 5.8$ & $23.2 / 26.6$ \\
\hline 6.84 & $1.9 / 2.2$ & $2.9 / 3.8$ & $14.0 / 15.9$ \\
\hline 10.3 & $1.5 / 1.8$ & $2.4 / 2.9$ & $10.3 / 11.7$ \\
\hline 27.4. & $0.9 / 0.9$ & $1.3 / 1.5$ & $4.9 / 5.6$ \\
\hline 150 & $0.3 / 0.3$ & $0.5 / 0.5$ & $1.5 / 1.6$ \\
\hline
\end{tabular}

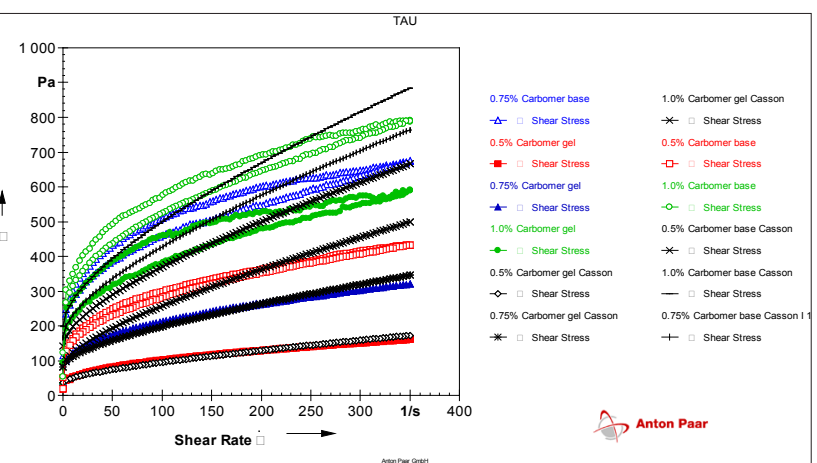

Figure 3.1. Experimental flow rheograms of carbomer gel bases and carbomer gels containing API and calculated (theoretical) flow rheograms according to the Casson model
Table 2.2. Calculated structural-mechanical indices of xanthan gum gel bases and API-loaded xanthan gum gels

\begin{tabular}{|c|c|c|c|}
\hline \multirow{2}{*}{ Indicators } & \multicolumn{3}{|c|}{ Gel bases } \\
\hline & $1.5 \%$ xanthan & $2.0 \%$ xanthan & $2.5 \%$ xanthan \\
\hline $\begin{array}{l}\text { Hysteresis loop } \\
\text { area, } \mathrm{Pa} / \mathrm{s}\end{array}$ & -4637.03 & -10275.08 & -10117.30 \\
\hline $\begin{array}{l}\text { The yield stress by } \\
\text { Casson } \mathrm{T}_{0}, \mathrm{~Pa}\end{array}$ & 7.51 & 27.97 & 89.11 \\
\hline $\begin{array}{l}\text { Viscosity at infinite } \\
\text { shear rate by } \\
\text { Casson } \eta_{\infty}, \mathrm{Pa} \cdot \mathrm{s}\end{array}$ & 0.23 & 0.37 & 0.48 \\
\hline $\begin{array}{l}\text { Dynamic } \\
\text { liquefaction } \\
\text { coefficient } \mathrm{K}_{\mathrm{d} 1}, \%\end{array}$ & 45.57 & 49.43 & 53.89 \\
\hline \multirow{3}{*}{$\begin{array}{l}\text { Dynamic } \\
\text { liquefaction } \\
\text { coefficient } \mathrm{K}_{\mathrm{d} 2}, \%\end{array}$} & 61.57 & 64.31 & 68.65 \\
\hline & \multicolumn{3}{|c|}{ API-loaded gels } \\
\hline & $1.5 \%$ xanthan & $2.0 \%$ xanthan & $2.5 \%$ xanthan \\
\hline $\begin{array}{l}\text { Hysteresis loop } \\
\text { area, } \mathrm{Pa} / \mathrm{s}\end{array}$ & -987.35 & -2354.97 & -4836.81 \\
\hline $\begin{array}{l}\text { The yield stress by } \\
\text { Casson } \mathrm{T}_{0}, \mathrm{~Pa}\end{array}$ & 4.01 & 7.46 & 64.65 \\
\hline $\begin{array}{l}\text { Viscosity at infinite } \\
\text { shear rate by } \\
\text { Casson } \eta_{\infty}, \mathrm{Pa} \cdot \mathrm{s}\end{array}$ & 0.16 & 0.22 & 0.31 \\
\hline $\begin{array}{l}\text { Dynamic } \\
\text { liquefaction } \\
\text { coefficient } \mathrm{K}_{\mathrm{d} 1} \% \%\end{array}$ & 44.93 & 45.73 & 55.60 \\
\hline $\begin{array}{l}\text { Dynamic } \\
\text { liquefaction } \\
\text { coefficient } \mathrm{K}_{\mathrm{d} 2} \% \%\end{array}$ & 62.63 & 63.88 & 70.50 \\
\hline
\end{tabular}

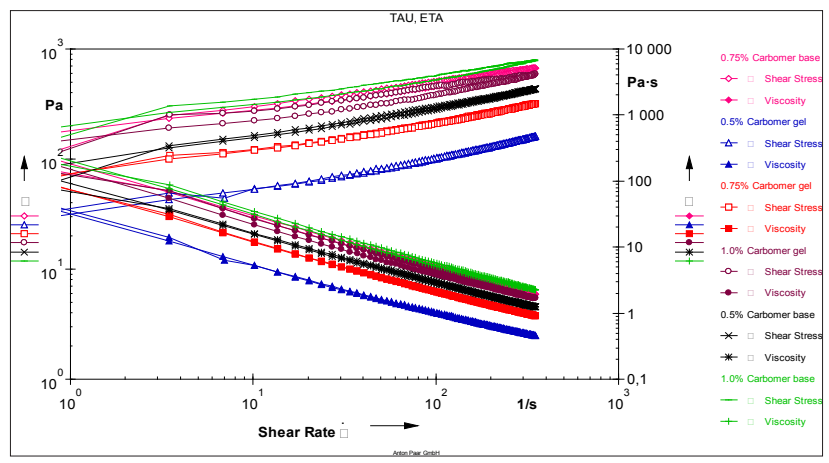

Figure 3.2. The dependence of the shear stress $(\tau, \mathrm{Pa})$ of gel carbomer bases and carbomer gels with API content on the shear rate gradient $\left(\dot{\gamma}, \mathrm{s}^{-1}\right)$ and the dependence of the structural viscosity $(\eta, \mathrm{Pa} \cdot \mathrm{s})$ on the shear rate gradient $\left(\dot{\gamma}, \mathrm{s}^{-1}\right)$ - logarithmic coordinates $(\log )$

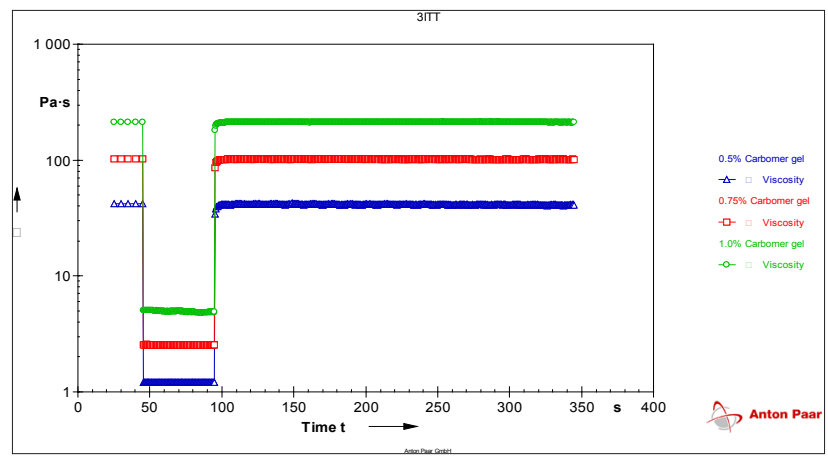

Figure 3.3. Dependence of structural viscosity $(\eta, P a \cdot s)$ of carbomer gels with API content on the shear rate gradient in the mode $1 \mathrm{~s}^{-1}-150 \mathrm{~s}^{-1}-1 \mathrm{~s}^{-1}$ 
Table 3.1. Values of shear stress and structural viscosity of carbomer gel bases and API-loaded carbomer gels in the order of increasing/decreasing shear rate gradient

\begin{tabular}{|c|c|c|c|}
\hline \multirow{2}{*}{$\begin{array}{l}\text { Shear rate gradient, } \\
\qquad\left(\dot{\mathrm{y}}, \mathrm{s}^{-1}\right)\end{array}$} & \multicolumn{3}{|c|}{ Gel bases } \\
\hline & $0.5 \%$ carbomer & $0.75 \%$ carbomer & $1.0 \%$ carbomer \\
\hline \multicolumn{4}{|c|}{$\begin{array}{l}\text { The shear stress }(\mathrm{T}, \mathrm{Pa}) \text { at increasing/ } \\
\text { decreasing the shear rate gradient }\left(\dot{\mathrm{Y}}, \mathrm{s}^{-1}\right)\end{array}$} \\
\hline 0.1 & $18.9 / 50.8$ & $38.6 / 113$ & $53.3 / 122$ \\
\hline 3.4 & $132 / 126$ & $248 / 234$ & $304 / 266$ \\
\hline 6.84 & $151 / 144$ & $278 / 260$ & $329 / 294$ \\
\hline 10.3 & $164 / 152$ & $300 / 277$ & $349 / 314$ \\
\hline 27.4 & 207/196 & $372 / 335$ & $429 / 380$ \\
\hline 150 & $335 / 322$ & $558 / 504$ & $640 / 589$ \\
\hline \multicolumn{4}{|c|}{$\begin{array}{c}\text { Structural viscosity }(n, P a \cdot s) \text { at increasing/ } \\
\text { decreasing shear rate gradient }\left(\dot{y}, s^{-1}\right)\end{array}$} \\
\hline 0.1 & $223 / 497$ & $413 / 1090$ & $592 / 1160$ \\
\hline 3.4 & $38.3 / 36.4$ & $71.7 / 67.7$ & $87.4 / 76.7$ \\
\hline 6.84 & $22.1 / 21.1$ & $40.7 / 37.1$ & $48.1 / 43.1$ \\
\hline 10.3 & $16.1 / 15.4$ & $29.5 / 27.2$ & $31.2 / 30.8$ \\
\hline 27.4 & $7.68 / 7.25$ & $13.8 / 12.4$ & $15.6 / 14.1$ \\
\hline \multirow[t]{3}{*}{150} & $2.21 / 2.13$ & $3.69 / 3.32$ & $4.22 / 3.89$ \\
\hline & \multicolumn{3}{|c|}{ API-loaded gels } \\
\hline & $0.5 \%$ carbomer & $0.75 \%$ carbomer & $1.0 \%$ carbomer \\
\hline \multicolumn{4}{|c|}{$\begin{array}{l}\text { The shear stress }(\mathrm{T}, \mathrm{Pa}) \text { at increasing/ } \\
\text { decreasing the shear rate gradient }\left(\dot{\hat{y}}, \mathbf{s}^{-1}\right)\end{array}$} \\
\hline 0.1 & $19.5 / 18.1$ & $35.7 / 42.3$ & $33.3 / 95.4$ \\
\hline 3.4 & $49.0 / 42.9$ & $108.0 / 101$ & 251.0/192.0 \\
\hline 6.84 & $44.4 / 49.2$ & $115.0 / 111$ & $261.0 / 210.0$ \\
\hline 10.3 & $53.6 / 53.3$ & $123.0 / 120.0$ & 272.0/223.0 \\
\hline 27.4 . & $66.5 / 68.9$ & $150.0 / 148.0$ & $331.0 / 269.0$ \\
\hline 150 & $117.0 / 119.0$ & $245.0 / 240.0$ & $499.0 / 438.0$ \\
\hline \multicolumn{4}{|c|}{$\begin{array}{c}\text { Structural viscosity }(n, P a \cdot s) \text { at increasing/ } \\
\text { decreasing shear rate gradient }\left(\dot{\gamma}, s^{-1}\right)\end{array}$} \\
\hline 0.1 & 199.0/179.0 & $356.0 / 415.0$ & $351.0 / 924.0$ \\
\hline 3.4 & $14.1 / 12.4$ & $30.9 / 29.1$ & $71.6 / 55.4$ \\
\hline 6.84 & $6.3 / 7.2$ & $16.8 / 16.3$ & $38.1 / 30.8$ \\
\hline 10.3 & $5.3 / 5.2$ & $12.0 / 11.7$ & $26.7 / 21.9$ \\
\hline 27.4 . & $2.5 / 2.6$ & $5.6 / 5.5$ & $12.3 / 9.9$ \\
\hline 150 & $0.6 / 0.8$ & $1.6 / 1.6$ & $3.2 / 2.8$ \\
\hline
\end{tabular}

\section{DISCUSSION}

As can be seen, all samples of gels, both blank and APIloaded, have a pseudoplastic flow, as the viscosity decreases with increasing shear force (Figures 1.2, 1.4, 2.2, 3.2), and the beginning of the flow is as close as possible to the point of intersection of the axes. A truly plastic type of flow or steady flow begins at an interval when the viscosity is directly proportional to the shear stress and inversely proportional to the shear rate gradient, that is, at any value of the shear stress the viscosity remains constant $(\eta=\tau / \dot{\gamma}[\mathrm{Pa} \cdot \mathrm{s}])$. The pseudoplastic type of flow is a priority for topical products applied to the skin and is the most common, indicating their ability to deform and easily spread over the skin $[3,24]$.

As can be seen from Figures 1.1, 1.2, 1.3, 1.4, 2.1, 2.2, 3.1 and 3.2 , the introduction of active substances significantly reduces the initial structural and mechanical parameters of
Table 3.2. Calculated structural-mechanical parameters of carbomer gel bases and API-loaded carbomer gels

\begin{tabular}{|c|c|c|c|}
\hline \multirow{2}{*}{ Indicators } & \multicolumn{3}{|c|}{ Gel bases } \\
\hline & $0.5 \%$ carbomer & $0.75 \%$ carbomer & $1.0 \%$ carbomer \\
\hline $\begin{array}{l}\text { Hysteresis loop } \\
\text { area, } \mathrm{Pa} / \mathrm{s}\end{array}$ & 3540.81 & 12923.34 & 14174.17 \\
\hline $\begin{array}{l}\text { The yield stress by } \\
\text { Casson } \mathrm{T}_{0}, \mathrm{~Pa}\end{array}$ & 77.17 & 160.35 & 191.39 \\
\hline $\begin{array}{l}\text { Viscosity at infinite } \\
\text { shear rate by } \\
\text { Casson } \eta_{\infty}, \mathrm{Pa} \cdot \mathrm{s}\end{array}$ & 0.52 & 0.64 & 0.72 \\
\hline $\begin{array}{l}\text { Dynamic } \\
\text { liquefaction } \\
\text { coefficient } \mathrm{K}_{\mathrm{d} 1}, \%\end{array}$ & 57.96 & 58.86 & 60.87 \\
\hline \multirow{3}{*}{$\begin{array}{l}\text { Dynamic } \\
\text { liquefaction } \\
\text { coefficient } \mathrm{K}_{\mathrm{d} 2}, \%\end{array}$} & 71.22 & 73.26 & 72.95 \\
\hline & \multicolumn{3}{|c|}{ API-loaded gels } \\
\hline & $0.5 \%$ carbomer & $0.75 \%$ carbomer & $1.0 \%$ carbomer \\
\hline $\begin{array}{l}\text { Hysteresis loop } \\
\text { area, } \mathrm{Pa} / \mathrm{s}\end{array}$ & -562.80 & 783.81 & 16472.13 \\
\hline $\begin{array}{l}\text { The yield stress by } \\
\text { Casson } \mathrm{T}_{0}, \mathrm{~Pa}\end{array}$ & 34.29 & 79.04 & 135.61 \\
\hline $\begin{array}{l}\text { Viscosity at infinite } \\
\text { shear rate by } \\
\text { Casson } \eta_{\infty}, \mathrm{Pa} \cdot \mathrm{s}\end{array}$ & 0.15 & 0.27 & 0.57 \\
\hline $\begin{array}{l}\text { Dynamic } \\
\text { liquefaction } \\
\text { coefficient } \mathrm{K}_{\mathrm{d} 1}, \%\end{array}$ & 62.77 & 61.17 & 62.71 \\
\hline $\begin{array}{l}\text { Dynamic } \\
\text { liquefaction } \\
\text { coefficient } \mathrm{K}_{\mathrm{d} 2} \% \%\end{array}$ & 69.39 & 71.53 & 73.82 \\
\hline
\end{tabular}

blank hydrogels, to which in addition to the gelling agent and water, $10 \%$ propylene glycol was added.

HPMC hydrogels have a pronounced fluidity ability, the yield stress $\tau 0$ of samples calculated by the mathematical model of Casson for gel bases is $0.81 \mathrm{~Pa}, 10.79 \mathrm{~Pa}$, 15.69 $\mathrm{Pa}$ and $26.87 \mathrm{~Pa}$, and for gels with API content it is $0.45 \mathrm{~Pa}, 9.22 \mathrm{~Pa}, 11.77 \mathrm{~Pa}$ and $13.38 \mathrm{~Pa}$, respectively, for samples with a concentration of HPMC of $1.2 \%, 1.6 \%$, $1.8 \%$ and $2.0 \%$, i.e. the flow of samples occurs arbitrarily and is HPMC-concentration-dependent. The yield stress is the stress that causes unlimited deformation at the moment when the sample begins to flow. The yield stress corresponds to the transition from elastic to plastic deformation or is the yield point below which the sample behaves as a solid. A study of HPMC hydrogels indicates that higher polymer concentrations give a higher resistance to the flow, even if the samples practically do not show the yield point.

Samples of HPMC hydrogels are highly thixotropic systems, as the structure is completely restored after the applied shear stress, as evidenced by the practical coincidence of the ascending and descending flow curves forming the hysteresis loop and a small area of the hysteresis loop itself (Table 1.2). At this, the restoration of the structure coincides in time with the liquefaction. Thixotropy of structured dispersed systems is manifested as liquefaction with sufficiently intense mixing and their thickening (solidification) after cessation of mechanical action $[25,26]$. Controlled shear rate test conditions (Figures 1.1, 2.1, 3.1) also allow estimating the thixotropy of hydrogels with a gradual decrease in shear rate.

At low values of shear rate $0.1 \mathrm{~s}^{-1}$, the samples exhibit rheopectic properties, characterized by a minor increase in viscosity (Figures 1.2, 1.4, 2.2, 3.2, Tables 1.1, 2.1, 3.1). True "rheopectic" properties are characteristic of dilatant dispersed systems, and are expressed in the increase of 
viscosity with increasing shear stress. At rest, such systems restore their initial properties, and the transition to a particular state depends on the time. Rheopectic dispersion systems can change infinitely from a state of increased viscosity with a prolonged deforming force, to a state of reduced viscosity at rest. In rheopectic liquids, in the CSR mode, there is an inverse arrangement of the flow curves (counterclockwise): the curve recorded at decreasing shear rate is above the curve obtained with increasing shear rate. For such systems, the calculated area of the hysteresis loop becomes negative, therefore, in the literature, rheopecty is referred to as negative thixotropy or antithixotropy [25-27].

The reason for the rheopecty is seen by some scientists in the fact that the parallel orientation of the elongated particles during the flow helps establishing bonds between them and thus promotes gel formation. Other scientists believe that the cause of rheopecty lies in the emergence of slight turbulence during the flow of a system - which accelerates the establishment of contact between the particles [28-31].

Hydrogels made on the basis of xanthan gum exhibit rheopectic properties throughout the shear rates range (Figure 2.2, Table 2.1). The calculated hysteresis loop area using RheoPlus software is represented in the negative value. The introduction of active substances dilutes blank hydrogels of xanthan gum, reducing the overall structural and mechanical properties. Thus, the yield points of xanthan gum gel bases $\tau_{0}$ of 7.15 $\mathrm{Pa}, 27.97 \mathrm{~Pa}$ and $89.11 \mathrm{~Pa}$ are reduced to 4.01 $\mathrm{Pa}, 7.46 \mathrm{~Pa}$ and $64.65 \mathrm{~Pa}$ for xanthan gum concentrations of $1.5 \%, 2.0 \%$ and $2.5 \%$, respectively (Figures 2.1, 2.2, Tables 2.2).

The most resistant to the applied deformation are carbomer-based hydrogels - the calculated yield points $\tau_{0}$, are 77.17 $\mathrm{Pa}, 160.35 \mathrm{~Pa}$ and 191.39 $\mathrm{Pa}$ for gel bases and 34.29 $\mathrm{Pa}, 79.04 \mathrm{~Pa}$ and $135.61 \mathrm{~Pa}$ for carbomer gels with API content for carbomer concentrations $0.5 \%, 0.75 \%$ and $1.0 \%$, respectively (Figure 3.2, Table 3.2). Conventionally, the shear stress interval from zero to the yield point in the CSR rheometers can be considered as holding elastic properties, therefore, such systems can be considered to be viscoelastic solids (Figure 3.1). In these, the higher the yield point value, the more resilient the system is. This allows indirectly asserting the bioadhesive properties of carbomer hydrogels. In the range of low shear rates, rheopectic behavior is observed in the API-loaded $0,5 \%$ carbomer gel sample, similarly to that of the HPMC hydrogels (Table 3.1).

In the literature, it is reported that in many cases, when the hysteresis of the flow curve is counter-clockwise, the material is not truly rheopectic. One of the reasons is the influence of the programmed rate of increase or decrease of the rotation speed of the rotor. Unless a sufficiently long period of time is provided to achieve an equilibrium state of interconnected shear rate and shear stress, the shear stress will start to increase even before the corresponding shear rate is reached. Therefore, in order to detect the true thixotropic or true rheopectic properties of API-loaded hydrogels, all samples were subjected to study at three intervals when in a state of controlled shear rate: rest (previous minimum shear, $1 \mathrm{~s}^{-1}$ ) - high shear (deformation of the system, $150 \mathrm{~s}^{-1}$ ) - relaxation (structure restoration $1 \mathrm{~s}^{-1}$ ). The results of the studies are shown in Figures 1.5, 2.3, 3.3. All samples of the studied hydrogels can be characterized as systems with highly thixotropic properties.

As it can be seen in Figures 1.5, 2.3 and 3.3, at low shear rates, the effect of the shear orientation is negligible, and all molecules or particles in the fluid carry out a chaotic Brownian motion. When the shear rate increases to $150 \mathrm{~s}^{-1}$, orientation of molecules or particles caused by it far outweighs the disorienting effect of Brownian motion, the viscosity of hydrogels decreases significantly and approaches the final constant $\eta_{\infty}$. Moving to even higher shear rates will not lead to a further decrease in viscosity, since optimal conditions for perfect particle orientation have already been achieved.

In the conditions of experimentation in a state of constant shear rate of $150 \mathrm{~s}^{-1}$ duration $50 \mathrm{~s}$, the value of structural viscosity for HPMC hydrogels obtained for concentrations of $1.2 \%, 1.6 \%, 1.8 \%$ and $2.0 \%$, respectively, are: 0.93 $\mathrm{Pa} \cdot \mathrm{s}, 1.9 \mathrm{~Pa} \cdot \mathrm{s}, 2.97 \mathrm{~Pa} \cdot \mathrm{s}$ and $3.43 \mathrm{~Pa} \cdot \mathrm{s}$ (Figure 1.5). For xanthan gum hydrogels, of concentrations $1.5 \%, 2.0 \%$ and $2.5 \%$, respectively, the corresponding values are 0.53 $\mathrm{Pa} \cdot \mathrm{s}, 0.81 \mathrm{~Pa} \cdot \mathrm{s}$ and $2.42 \mathrm{~Pa} \cdot \mathrm{s}$ (Figure 2.3). For carbomer hydrogels of concentrations $0.5 \%, 0.75 \%$ and $1.0 \%$, respectively, the corresponding values are $1.2 \mathrm{~Pa} \mathrm{~s}, 2.54 \mathrm{~Pa} \cdot \mathrm{s}$ and $4.9 \mathrm{~Pa} \cdot \mathrm{s}$ (Figure 3.3). Under these conditions, all the hydrogels recovered by $100 \%$ within 5 minutes, confirming the stability of the systems under study. This guarantees the restoration of the structure after the manufacturing process.

Mechanical liquefaction coefficients are often used to estimate the degree of mechanical destruction [32]. Here, $\mathrm{K} . \mathrm{d} 1$ is calculated in the range of velocities that correspond to the rate of application to the surface when using the medicine, and $\mathrm{K}_{\mathrm{d} 2}$ - to the speed of technological process of production. It is natural that $\mathrm{K}_{{ }_{\mathrm{d} 2}}>\mathrm{K}_{\mathrm{d}_{1}}$, since the systems are more liquid at high strain rates. The regularity of the increase in $\mathrm{K}_{\mathrm{d} 1}$ and $\mathrm{K}_{\mathrm{d} 2}$ coefficients is observed with increasing concentration of the gelling agent.

\section{CONCLUSIONS}

Based on rheological studies of hydrogels containing $0.1 \%$ dimethindene maleate and $3.0 \%$ dexpanthenol made on the basis of HMPC, xanthan gum and carbomer, it is recommended after performing the test of the dynamics of release under conditions in vitro, and using samples of HPMC hydrogels $1.8 \%$ and $2.0 \%$, xanthan gum hydrogels $2.0 \%$ and $2.5 \%$, carbomer hydrogels $0.5 \%$ and $0.75 \%$, that the use of one of these formulations as a final composition provides sufficient adhesion during application and will not complicate the scaling-up of the process.

In contrast, samples of HPMC hydrogels $1.2 \%$ and $1.6 \%$ and xanthan gum hydrogels of $1.5 \%$ have low structural and mechanical properties, and have an arbitrary flow and do not provide effective adhesion. Moreover, the sample of carbomer hydrogel at $1.0 \%$ has high structural and mechanical parameters, and such a concentration is not rational from an economic point of view.

\section{CONFLICT OF INTERESTS}

The authors declare that they have no conflicts of interest. 


\section{ORCID iDs}

Tetiana V. Popova (Dhttps://orcid.org/0000-0003-2334-903X Halyna P. Kukhtenko (1) https://orcid.org/0000-0002-7914-8053 Ievgenii V. Gladukh (Dhttps://orcid.org/0000-0002-5739-9257 Oleksandr S. Kukhtenko (1) https://orcid.org/0000-0003-4908-6717 Anzhela B. Olkhovska (Dhttps://orcid.org/0000-0002-0237-5741

\section{REFERNCES}

1. Medicines. Pharmaceutical development (ICH Q8) Kyiv; 2011. ST-N MoHU 42-3.0:2011.

2. Baudonnet L, Pere D, Michaud P, Grossiord L, Rodriguez F. Effect of dispersion stirring speed on the particle size distribution and rheological properties of carbomer dispersions and gels. J Dispers Sci Technol. 2002;23(4):499-510.

3. Mazger TG. The Rheology Handbook: For users of rotational and oscillatory rheometers. 2 nd revised ed. Hannover:Vincentz Network; 2006:298.

4. Eschler D, Klein P. An evidence-based review of the efficacy of topical antihistamines in the relief of pruritus. J Drugs Dermatol. 2010;9(8):992-7.

5. Proksch E, de Bony R, Trapp S, Boudon S. Topical use of dexpanthenol: a 70th anniversary article. J Dermatol Treat. 2017;28(8):766-73.

6. Guideline "Medicines. Bioequivalence study". State Enterprise "State Expert Center of the Ministry of Health of Ukraine". ST-N MoHU 42-7.2:2018. [Internet]. [cited 2021 Jan 05]. Available from: https:// zakon.rada.gov.ua/rada/show/v0022282-17/stru

7. Ministry of Health of Ukraine (n.d.). State Register of Medicines of Ukraine. Available from: http://www.drlz.kiev.ua [cited 2021 Jan 20].

8. Baek J, Lim J, Kang J, Shin S, Jung S, Cho C. Enhanced transdermal drug delivery of zaltoprofen using a novel formulation. Int J Pharm. 2013;453(2):358-62.

9. Hamed R, AbuRezeq A, Tarawneh O. Development of hydrogels, oleogels, and bigels as local drug delivery systems for periodontitis. Drug Dev Ind Pharm. 2018;44(9):1488-97.

10. Carvalho F, Calixto G, Hatakeyama I, Luz GM, Gremiao M, Chorilli M.. Rheological, mechanical, and bioadhesive behavior of hydrogels to optimize skin delivery systems. Drug Dev Ind Pharm. 2013;39(11):1750-7.

11. Sandolo C, Coviello T, Matricardi P, Alhaique F. Characterization of polysaccharide hydrogels for modified drug delivery. Eur Bioph $J$ Biophy. 2007;36(7):693-700.

12. Anurova MN, Bakhrushina EO, Demina NB. A review of modern gel-forming agents in technology of dosage Forms. Khimikofarmatsevticheskii zhurnal. 2015;49(9):39-46.

13. Sosnik A, Seremeta KP. Polymeric hydrogels as technology platform for Drug Delivery Applications. Gels. 2017;3(3).

14. Stokes J, Frith W. Rheology of gelling and yielding soft matter systems. Soft Matter. 2008;4(6):1133-40.
15. Pisal P, Patil S, Pokharkar V. Rheological investigation and its correlation with permeability coefficient of drug loaded carbopol gel: influence of absorption enhancers. Drug Dev Ind Pharm. 2013;39(4):593-9.

16. Cintra G, Pinto L, Calixto G, Soares C, Von Zuben E, Scarpa M, et al. Bioadhesive surfactant systems for Methotrexate skin delivery. Molecules. 2016;21(2).

17. Sheskey PJ, Cook WG, Cable CG. Handbook of Pharmaceutical Excipients. 8th ed. London: American Pharmacists Association, Pharmaceutical Press; 2017:1216.

18. Dinkgreve M, Fazilati M, Denn M, Bonn D. Carbopol: From a simple to a thixotropic yield stress. J Rheol. 2018;62(3):773-80.

19. Kukhtenko H, Gladukh I, Kukhtenko O, Soldatov D. Influence of excipients on the structural and mechanical properties of semisolid dosage forms. AJP. 2017;11(3):575-8.

20. Lilienblum W, Bernauer U, Bodin L, Celleno L, Chaudhry Q, Coenraads P, et al. Opinion of the Scientific Committee on Consumer Safety (SCCS) - Final version of the opinion on Phenoxyethanol in cosmetic products. Regulato Toxicol Pharmacol. 2016;82:156.

21. Lyapunov AN, Bezuglaya EP, Lyapunov NA, Kirilyuk IA. Studies of carbomer gels using rotational viscometry and spin probes. Pharm Chem J. 2015;49(9):639-44.

22. Islam M, Rodriguez-Hornedo N, Ciotti S, Ackermann C. Rheological characterization of topical carbomer gels neutralized to different $\mathrm{pH}$. Pharm Res. 2004;21(7):1192-9.

23. Mallia V, Weiss R. Correlations between thixotropic and structural properties of molecular gels with crystalline networks. Soft Matter. 2016;12(16):3665-76.

24. Malkin AYa, Isaev AI. Rheology: concepts, methods, applications. trans. from Eng. St. Petersburg: Profession; 2007:560.

25. Ong E, O'Byrne S, Liow J. Yield stress measurement of a thixotropic colloid. Rheologica Acta. 2019;58(6-7):383-401.

26. Mendes P, Thompson R. A unified approach to model elastoviscoplastic thixotropic yield-stress materials and apparent yieldstress fluids. Rheologica Acta. 2013;52(7):673-94.

27. Bautista F, de Santos J, Puig J, Manero O. Understanding thixotropic and antithixotropic behavior of viscoelastic micellar solutions and liquid crystalline dispersions. I. The model. J Newtonian Fluid Mech. 1999;80(2-3):93-113

28. Buitenhuis J, Ponitsch M. Negative thixotropy of polymer solutions. 1. A model explaining time-dependent viscosity. Colloid and Polymer Science. 2003;281(3):253-9.

29. Buitenhuis J, Springer J. Negative thixotropy of polymer solutions. 2. A systematic study of the time-dependent viscosity of partially hydrolyzed polyacrylamide. Colloid Polymer Sci. 2003;281(3):260-6.

30. Masalova I, Taylor M, Kharatiyan E, Malkin AY. Rheopexy in highly concentrated emulsions. J Rheol. 2005;49(4):839-49.

31. Postoy V, Kukhtenko H, Vyshnevska L, Gladukh I, Semchenko K. Study of rheological behaviour of hydroxyethyl cellulose gels in the development of the composition and technology of the medicine with anti-inflammatory activity. Pharmacia. 2019;66(4):187-92.

32. Gladukh I, Grubnik I, Kukhtenko H. Structural-mechanical studies of phytogel “Zhivitan”. JPSR. 2017;9(9):1672-76. 